\title{
Results of $\mathrm{CO}_{2}$ laser-assisted sclerotomy surgery (CLASS procedures) in eyes with primary open-angle glaucoma
}

\author{
ZZ NAGY*, M BAUSZ, K KRÁNITZ and HJ KISS
}

Department of Ophthalmology, Semmelweis University, Budapest, Hungary

(Received: October 10, 2018; revised manuscript received: November 30, 2018; accepted: December 13, 2018)

\begin{abstract}
Purpose: This study aimed to examine the effectiveness of $\mathrm{CO}_{2}$ laser-assisted sclerectomy surgery (CLASS) in eyes with primary open-angle glaucoma (POAG) showing progression in spite of maximal local antiglaucomatous therapy. Materials and methods: Patients with progressive POAG received CLASS treatment. We performed CLASS on 15 eyes (eight males and seven females). The primary endpoint was the change in the intraocular pressure (IOP), and additionally best spectacle-corrected visual acuity (BSCVA), C/D ratio (cup-to-disc), as well as use of antiglaucomatous drops were also investigated. Following the preoperative assessment, measurements were performed at 6-month followup. Results: The average preoperative IOP was $26.13 \pm 6.79 \mathrm{mmHg}$ that dropped to $9.57 \pm 4.09 \mathrm{mmHg}$ at 1 day. IOP was stable at 1 month, 3 months, and 6 months. The BSCVA decreased to the 1-day and 1-week follow-up but returned to its original value to the 1-month check-up. Preoperatively, all patients were on maximal antiglaucoma drop therapy, after CLASS none of the patients needed antiglaucomatous treatment at 1 month. However, at 3 months, one of them needed antiglaucoma drops. C/D ratio showed non-significant changes. Conclusions: CLASS procedure was found to be effective in decreasing IOP in POAG patients whose IOP was not compensated with maximal antiglaucomatous local therapy; patients needed significantly less local therapy following the $\mathrm{CO}_{2}$ laser surgery.
\end{abstract}

Keywords: glaucoma, deep sclerectomy, $\mathrm{CO}_{2}$ laser, CLASS, glaucoma surgery

\section{INTRODUCTION}

Primary open-angle glaucoma (POAG) is a progressive, chronic disease, which is characterised by gradual increase of the intraocular pressure (IOP) and damage of the small arterioles of the optic nerve, with a consecutive imbalance between IOP and circulation of the optic nerve head. Consequently, the ophthalmologist may observe cupping of the optic nerve head together with visual field defect.

Undiagnosed POAG is relatively frequent in the Caucasian population and the disease may remain hidden until significant visual field defect. The problem is that this defect is irreversible; therefore, early diagnosis and efficient treatments have utmost importance halting the visual field deterioration and protecting visual acuity.

The cause of POAG is multifactorial with family history certainly playing an important role in it. Usually, the resistance of the trabecular meshwork is increased; therefore, the aqueous humour produced by the ciliary processes cannot leave the eye properly through the trabecular meshwork and consequently the IOP increases. The increase is usually gradual and affects the two eyes differently, so sometimes the patient does not notice it, because they do not test their eyes (the visual acuity and visual field) with separate eyes. Conservative therapy means using eye drops in order to decrease the IOP. Several eyedrops are available in Europe, but in some patients conservative therapy cannot decrease
IOP below abnormal values (usually below $21 \mathrm{mmHg}$ ) even with maximal therapy. For these patients, surgery is needed. Before antiglaucomatous eyedrops, trabeculectomy was the most frequently performed IOP-lowering surgical intervention, although there were several side effects, including postoperative hypotony, choroidal detachment, suprachoroidal bleeding, endophthalmitis, malignant glaucoma, and cataract [1]. Therefore, non-penetrating glaucoma surgery came into focus a decade ago [2].

Performing non-penetrating surgery manually is challenging and difficult, and sometimes around the last step of the surgery, the thin sclera perforates and the surgery is transformed into a penetrating procedure with the all possible side effects.

Manual deep sclerectomy, according to the data published in literature [2-5], results in less reduction of IOP, and similarly the side effects are also significantly milder. The reason is that the trabeculo-Descemet membrane stays intact, so the decrease of IOP is more gradual in these patients.

Because manual non-penetrating glaucoma surgery is difficult, $\mathrm{CO}_{2}$ laser has been introduced to standardise the method and to increase predictability and safety. The new

\footnotetext{
* Corresponding author: Zoltán Zsolt Nagy, MD, PhD, DSc; Department of Ophthalmology, Semmelweis University, Budapest, Hungary; Phone: +36 1303 9435; E-mail: nagy.zoltan_zsolt@med. semmelweis-univ.hu
}

This is an open-access article distributed under the terms of the Creative Commons Attribution-NonCommercial 4.0 International License, which permits unrestricted use, distribution, and reproduction in any medium for non-commercial purposes, provided the original author and source are credited, a link to the CC License is provided, and changes - if any - are indicated. 
technique was first introduced and published by Assia et al. [6]. Several technical modifications have been made since the first prototype, and by now the $\mathrm{CO}_{2}$ laser is available commercially $[3,4,7]$. In this article, we present the first clinical results with $\mathrm{CO}_{2}$ laser-assisted sclerectomy surgery (CLASS).

\section{MATERIALS AND METHODS}

CLASS procedure was performed in 15 POAG eyes in the Department of Ophthalmology, Semmelweis University, Budapest, Hungary. Patients with progressive POAG glaucoma received CLASS treatment on the worse eye. Fifteen eyes of 14 patients were enrolled into the study. The average age of the patients was $62.97 \pm 11.81$ years. Among them, eight were males and seven were females. During preoperative assessment, medical and family histories were recorded, IOP, as well as uncorrected and best spectacle-corrected visual acuity (UCVA and BSCVA) were determined, and $\mathrm{C} / \mathrm{D}$ ratio was documented. Similar measurements were performed during the follow-up visits at 1 day, 1 week, 1 month, 3 months, and 6 months postoperatively.

\section{Surgical method}

Most of the surgeries were performed under retrobulbar anesthesia, but in four cases, with less compliant patients, general anesthesia was applied. Fornix-based conjunctival flap was created at the beginning of the surgery, then a limbus-based $5 \times 5 \mathrm{~mm}$ scleral flap was made with a special single-use crescent knife (scleral flap was $1 \times 1 \mathrm{~mm}$ larger than in normal trabeculectomy procedures). Afterwards, flap preparation of $0.02 \%$ mitomycin-C (MMC) soaked using small sponges was tucked between the conjunctiva and the scleral flap for $2 \mathrm{~min}$. Then, the MMC was washed out and the $\mathrm{CO}_{2}$ laser was applied (IOPtima Ltd., Ramat-Gan, Israel). During the laser procedure, at first, a $4.0 \times 1.6 \mathrm{~mm}$ aqueous reservoir was created, then around the Schlemmchannel, another $4.0 \times 1.0 \mathrm{~mm}$ semiperforating scleral pool was created by the laser in a crescent-shaped form.

When bubble formation in the aqueous humour occurred, $\mathrm{CO}_{2}$ laser treatment was finished. The corners of the scleral flap were sutured with 10/0 nylon sutures and then the conjunctiva was also sutured with the $10 / 0$ nylon, especially at the edges. At the end of the surgery, subconjunctival dexamethasone was administered.

Patients were examined at 1 day, 1 week, 1 month, 3 months, and 6 months. If it was necessary, the conjunctival flap was checked more frequently (every week during the first postoperative month, then according to the protocol). UCVA and BSCVA were tested, IOP was measured by Goldmann tonometry, C/D ratio was determined, and the necessary antiglaucomatous drops were registered in the patient's file. Intraoperative and postoperative complications were also recorded and later analysed.

Statistical analysis was performed using the IBM SPSS Statistics for Windows v21 (IBM Corp., Released 2012, Armonk, NY, USA). Preoperative and postoperative IOP values and visual acuity results were compared with paired $t$-test.

\section{RESULTS}

The average preoperative IOP was $26.13 \pm 6.79 \mathrm{mmHg}$. Following the surgery, the IOP dropped already at Day 1 to $9.57 \pm 4.09 \mathrm{mmHg}(p<.001)$. The postoperative IOP was stable and significantly lower following the CLASS procedures $(p<.05)$ than the preoperative values on each followup visit (1 week, 1 month, 3 months, and 6 months; Figure 1). With regard to IOP, there was no significant difference between the result of the 6-month follow-up.

BSCVA decreased significantly on the first day following the surgery $(p=.017)$, improved significantly by the 1 -week check-up $(p=.039)$, and returned to the original value at the 1 -month visit $(p<.001)$. During the follow-up period, there was no significant difference between the preand postoperative BSCVA results 1 week after the surgery $(p>.05$; Figure 2)

The preoperative $\mathrm{C} / \mathrm{D}$ ratio was $0.84 \pm 0.17$, with no significant change during the follow-up period $(p>.05)$.

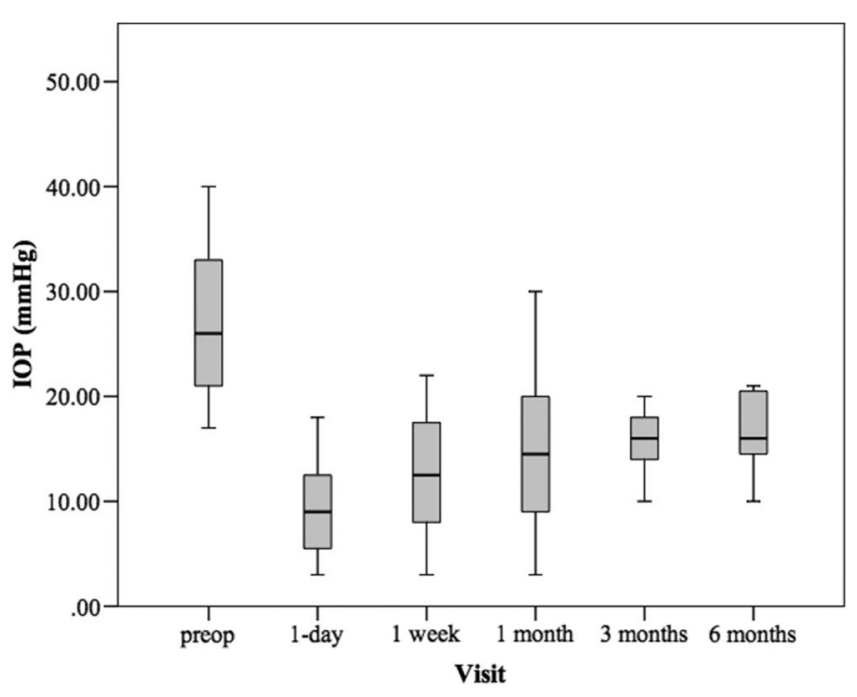

Figure 1. Changes of intraocular pressure (IOP) due to CLASS procedure

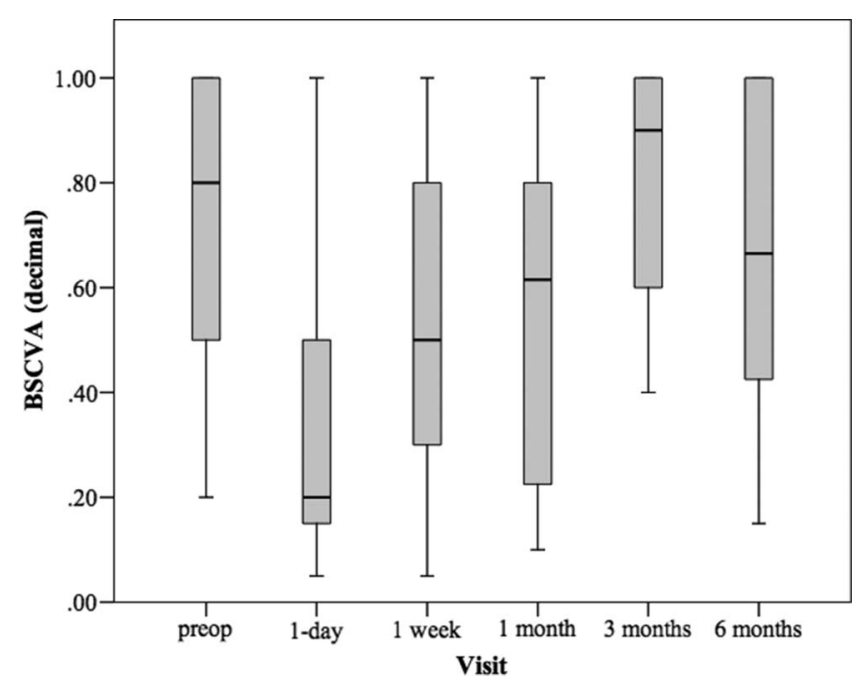

Figure 2. Changes of best-corrected visual acuity (BSCVA) due to CLASS procedure 
Preoperatively, all patients were on antiglaucomatous drop therapy, most of them using two or more types of drops. One patient was consuming oral acetazolamide (250 mg) pills; after CLASS procedure, they did not need it anymore. None of the patients needed antiglaucomatous treatment at 1 month; however, at 3 months, three of them needed one type of drop therapy. During the first month, one of the patients showed an IOP higher than $18 \mathrm{mmHg}$. Therefore, the decrease of drop therapy was significantly smaller compared to preoperative antiglaucoma drop use.

Among the patients, two had inadvertent scleral penetration during surgery; therefore, one case was converted into trabeculectomy and the other had a small injury of the ciliary body. No hyphaema or hypotony was experienced in these two cases, and IOP stayed within normal range during the whole follow-up period. On the other hand, in another patient, a transient choroidal detachment was found during the early postoperative period with lower IOP. This was resolved within 3 weeks and the IOP was in normal range (below $18 \mathrm{mmHg}$ ). In three cases, conjunctival vessel dilation and tortuosity were found without IOP rise.

\section{DISCUSSION}

The IOP decreasing effect is smaller in case of nonpenetrating glaucoma surgery, including the CLASS procedure as well, although severe postoperative complications are also much less common compared to filtration procedures $[1,5]$. Due to the milder IOP decrease, the danger of abrupt visual field loss is also rare, which can be experienced in advanced glaucoma cases following filtrating surgery, such as trabeculectomy.

CLASS offers a reasonable solution for the technical problems of manual non-penetrating surgeries as well, because this is a titratable method, the juxtacanalicular trabecular meshwork can be opened without a real perforation $[3,4,7]$. The aqueous humour appearing in the ablated area prevents further laser tissue destruction. When the underlying sclera is very thin, inadvertent scleral perforation may occur; however, with meticulous scleral flap preparation (not too thick), this complication can be avoided. In one case, we could not explain the cause of choroidal detachment, because there was no complete scleral perforation and the scleral flap was also not too thick. The $\mathrm{CO}_{2}$ laser is able to deliver even and planned size of tissue ablation; the surrounding tissue the thermal damage was minimal.

Using the MMC sponge, the chance of scarring, tissue fibrosis, and adhesion are significantly smaller.

In POAG cases with CLASS procedure in the literature, different results are published, in which some of the authors achieved a $45.1 \%$, others only $19.0 \%$ decrease of IOP during the 6-month follow-up period $[3,4,7]$.

Among our patients, IOP decrease was significant in patients who were on maximal antiglaucomatous topical therapy and showing glaucoma progression. Importantly, IOP stayed in the normal range; BSCVA did not decrease statistically in a significant way. During the first postoperative month, none of the patients needed antiglaucoma therapy, at months 3 and 6; three patients needed extra eye pressure-lowering drops (but only one type of drop).

Based on our results, the CLASS procedure was found to be effective and efficient in decreasing IOP in patients whose IOP was not compensated with maximal antiglaucomatous local therapy, and patients needed significantly less local therapy following the $\mathrm{CO}_{2}$ laser surgery.

\section{CONCLUSIONS}

We recommend the method in case of POAG. Possibly, the CLASS procedure can be applied in earlier glaucoma cases also to prevent serious progression and visual field deterioration. Nevertheless, we find it necessary to include a larger patient group with longer follow-up period to find out the real efficiency of the method.

Authors' contribution: ZZN performed surgery and collected data, finalised the manuscript. MB, KK, and HJK collected the data. HJK performed statistical analysis and finalised the text.

Ethical approval: All procedures were in accordance with the ethical standards of the institutional and/or national research committee and with the 1964 Helsinki Declaration and its later amendments or comparable ethical standards. Participants received both oral and written information about the study and they signed an informed consent form.

Conflict of Interest/Funding: The authors declare no conflict of interest and no financial support was received for this study.

\section{REFERENCES}

1. Eldaly MA, Bunce C, Elsheikha OZ, Wormald R. Nonpenetrating filtration surgery versus trabeculectomy for openangle glaucoma. Cochrane Database Syst Rev. 2014;2014(2): CD007059.

2. Gáspár Á, Sebestyén M, Salacz G. Tapasztalataink mély sclerectomiával glaucomás szemeken [Experiences with deep sclerectomy in glaucoma patients]. Ophthalmol Hung. 2005; 141(1):23-7.

3. Sohajda Z, Káldi I, Kiss M, Facskó A. CO2-lézerrel végzett mélysclerectomiával szerzett tapasztalatok [Experiences with CO2 laser-assisted sclerectomy surgery]. Orv Hetil. 2017; 158(18):701-5.

4. Skaat A, Goldenfeld M, Cotlear D, Melamed S. $\mathrm{CO}_{2}$ laserassisted deep sclerectomy in glaucoma patients. J Glaucoma. 2014;23(3):179-84.

5. Roy S, Mermoud A. Deep sclerectomy. Dev Ophthalmol. 2017;59:36-42. 
6. Assia EI, Rotenstreich Y, Barequet IS, Apple DJ, Rosner M, Belkin M. Experimental studies on nonpenetrating filtration surgery using the $\mathrm{CO}_{2}$ laser. Graefes Arch Clin Exp Ophthalmol. 2007;245(6):847-54.
7. Geffen N, Mimouni M, Sherwood M, Assia EI. Mid-term clinical results of $\mathrm{CO}_{2}$ laser-assisted sclerectomy surgery (CLASS) for open-angle glaucoma treatment. J Glaucoma. 2016;25(12):946-51. 